\title{
Predictores de las prácticas sobre el autoexamen de mamas en estudiantes de Enfermería de una universidad privada, Lima, Perú.
}

\author{
Katherine Jenny Ortiz Romaní ${ }^{1}$, María Celeste Gonzales Paredes ${ }^{2}$, Yonathan Josué Ortiz Romaní ${ }^{3}$
}

\footnotetext{
${ }^{1}$ Licenciada en Enfermería, Universidad Católica Sedes Sapientiae, Investigadora del Programa de Estudios de Enfermería, Lima, Perú. ORCID: 0000-0003-0331-4446

2 Estudiante de Pregrado en Enfermería, Universidad Católica Sedes Sapientiae, Programa de Estudios de Enfermería, Lima, Perú. ORCID:0000-0002-0371-0134

${ }^{3}$ Licenciada en Enfermería, Universidad Católica Sedes Sapientiae, Coordinador del Departamento de Investigación de la Facultad de Ciencias de la Salud, Investigador del Programa de estudios de Enfermería, Lima, Perú. ORCID:0000-0002-7678-8303.
}

Información del artículo

Recibido: 04-11-2020

Aceptado: 03-05-2021

DOI:

10.15517/revenf.v0i41.44466

\section{Correspondencia}

Katherine Jenny Ortiz

Romaní

Investigadora del Programa de Estudios de Enfermería, Universidad Católica Sedes Sapientiae, Lima, Perú

Email: kortiz@ucss.edu.pe

\section{RESUMEN}

Objetivo: El objetivo del estudio fue analizar los predictores de las prácticas sobre el autoexamen de mamas en estudiantes de Enfermería de la universidad Católica Sedes Sapientiae, Lima, Perú, 2019.

Metodología: Es un estudio observacional, analítico y transversal. La población estuvo conformada por 92 personas universitarias de ambos sexos. Se ejecutó una guía de evaluación para medir la técnica del autoexamen de mamas, un cuestionario autodirigido para evaluar el conocimiento de esta técnica y por último una ficha de datos para la variable realización de autoexamen de mamas, entre otras variables sociodemográficas y de formación académica.

Resultados: Entre los resultados principales, se muestra que el $65.22 \%$ de las personas estudiantes no realizó el autoexamen de mamas y el promedio de la técnica fue bajo ( $M=4.41)$ de un rango de 0 a 12 puntos. Asimismo, se evidenció que los predictores de la realización del autoexamen de mamas fueron el nivel alto de conocimiento de esta técnica (PR: 8.73; $p=0.022$ ), la edad (PR: $1.04 ; p=$ 0.021 ) y capacitación (PR: $2.42 ; p=0.005)$. Además, los predictores de la técnica fueron el conocimiento de esta (coeficiente: 1.70; $p=0.023$ ) y la edad (coeficiente: $1.70 ; p=0.023$ ).

Conclusión: En conclusión, la mayoría de estudiantes no realiza las prácticas sobre el autoexamen de mamas, además, los predictores de estas prácticas son el conocimiento sobre la técnica la edad y la capacitación. Ante estos hallazgos se deberían brindar intervenciones educativas promocionales y preventivas al estudiantado.

Palabras claves: Autoexamen-de-Mamas; Estudiantes-de-Enfermería;

Prevención-Primaria 


\section{ABSTRACT \\ Predictors of breast self-examination practices in nursing students from a private university, Lima, Peru.}

Aim: The objective of this study was to analyze the predictors of breast selfexamination practices of nursing students at the Catholic Sedes Sapientiae University, Lima, Peru, 2019.

Methods: It was an observational, analytical and cross-sectional study. The target population were 92 university students of both sexes. In order to analyze this, the researchers passed an evaluation guide to measure the breast selfexamination technique, a self-directed questionnaire to assess this technique's knowledge, and a data sheet for the variable performance of breast selfexamination, among other sociodemographic and academic training variables. Results: Part of the main results show that $65.22 \%$ of the students did not perform breast self-examinations, and the average of the technique was low with a range of 0 to 12 points $(M=4.41)$. The results also evidenced that the predictors of the performance of the breast self-examination were the high level of knowledge of this technique (PR: 8.73; $p=0.022$ ), the age (PR: 1.04; $p=0.021$ ), and the training (PR: 2.42; $p=0.005$ ). The predictors of the technique were the knowledge about it (coefficient: 1.70; $p=0.023$ ) and the age (coefficient: 1.70; $\mathrm{p}=0.023$ ).

Conclusion: In conclusion, the majority of students did not carry out the practices on breast self-examination; likewise, the predictors of these practices are the knowledge about this technique, the age, and the training. Given these findings, educational, promotional, and preventive interventions should be provided to the students.

Keywords: Breast Self-Examination; Students, Nursing; Primary-Prevention.

\section{RESUMO}

\section{Preditores de práticas de autoexame das mamas em estudantes de enfermagem de uma universidade privada, Lima, Peru}

Objetivo: O objetivo do estudo foi analisar os preditores das práticas de autoexame das mamas em estudantes de enfermagem da Universidade Católica Sedes Sapientiae, Lima, Peru, 2019.

Metodologia: É um estudo observacional, analítico e transversal; a população era composta por 92 universitários de ambos os sexos. Foi executado um roteiro de avaliação para mensurar a técnica do autoexame das mamas, um questionário autodirigido para avaliar o conhecimento dessa técnica e, por fim, uma planilha de dados da variável realização do autoexame das mamas entre outras variáveis sociodemográficas e de treinamento acadêmico. 
Resultados: Dentre os principais resultados, evidencia-se que $65.22 \%$ das alunas não realizaram o autoexame das mamas e a média da técnica foi baixa $(\mathrm{M}=4.41)$ com variação de 0 a 12 pontos, além disso, constatou-se que os preditores da realização do autoexame das mamas foram o alto nível de conhecimento desta técnica (RP: 8.73; $p=0.022$ ), idade (RP: $1.04 ; p=0,021$ ) e treinamento (RP: 2.42; $p=0.005$ ); e os preditores da técnica foram o conhecimento (coeficiente: $1.70 ; p=0.023$ ) e idade (coeficiente: $1.70 ; p=0.023$ ). Conclusão: Em conclusão, a maioria das alunas não realiza as práticas sobre o autoexame das mamas, da mesma forma, os preditores dessas práticas são o conhecimento dessa técnica; idade e treinamento. Diante desses achados, intervenções educacionais, promocionais e preventivas devem ser fornecidas aos alunos.

Palavras chave: Autoexame-de-Mama; Estudantes-de-Enfermagem; PrevençãoPrimária

\section{INTRODUCCIÓN}

El cáncer de mama es una enfermedad frecuente que afecta mayormente a la mujer. Esta patología puede ser tratada y diagnosticada a tiempo si se hace la promoción de la salud y prevención de la enfermedad, por parte de profesionales sanitarios, como lo son el personal de enfermería ${ }^{1,2}$. En América Latina y el Caribe, el cáncer de mama es el tipo de cáncer más común entre las mujeres y la segunda causa de muerte. Cada año en América, alrededor de 462000 mujeres son diagnosticadas con cáncer de mama y cerca de 100000 mueren debido a esta enfermedad. Si esta tendencia continúa, se estima que para el año 2030 el número de diagnósticos de esta patología puede aumentar en un $34 \%$ para esta región ${ }^{1}$. En el Perú, según el Instituto Nacional de Enfermedades Neoplásicas (INEN), el cáncer de mama ocupa el segundo lugar de todos los cánceres de la mujer. Asimismo, en el año 2018 se registraron 1379 casos de mujeres diagnosticadas con cáncer de mama, con lo cual se evidencia el aumento por año del número de diagnósticos de esta enfermedad a nivel nacional ${ }^{3}$.
Existen tres métodos de tamizaje para el cáncer de mama: mamografía, examen clínico de las mamas y el autoexamen de mamas (AEM), que es el método inicial $^{4,5}$. Referente a lo anterior, algunos estudios han demostrado que poseer un alto conocimientos del AEM es un predictor para realizar este examen evaluativo y que el tener entrenamiento o instrucción previa sobre el AEM se relaciona con el conocimiento y realización de esta práctica ${ }^{6,7,8,9,10}$. Asimismo, la literatura evidencia que, en el Perú, el $57.14 \%$ de todas las personas universitarias en Enfermería tuvo un conocimiento regular de esta técnica, el $26.4 \%$ no realizó el AEM y el $52.10 \%$ no aplicó la técnica ${ }^{11}$. Sin embargo, en estudios internacionales, centrados en población femenina, se encontró que gran porcentaje de las estudiantes presentaron un conocimiento excelente y prácticas adecuadas sobre el AEM $^{12,13}$.

Existe poca cantidad de estudios en el Perú y Latinoamérica relacionados al AEM, cuando se menciona la conciencia de prevención, se enfoca en estudiantes de la carrera de Enfermería y los pocos lugares donde se informan los resultados son pobres. Ante el aumento del cáncer de mama en el Perú, 
particularmente en mujeres ${ }^{3}$, es necesario mencionar que la practica correcta del AEM aumenta las probabilidades de detectar un nódulo en la etapa inicial, con lo que incrementan las posibilidades de supervivencia. Además, se considera un procedimiento simple, económico y no invasivo que ayuda a las mujeres a conocer sus senos, por lo cual es más probable que busquen atención médica, una mamografía o el examen clínico de las mamas, cuando surja la necesidad. En países con recursos limitados y en el Perú debe ser promovido el AEM tanto para la detección temprana del cáncer de mama como para prevenir morbilidades y mortalidades. La conciencia de la prevención del cáncer de mama no está bien documentada en la enseñanza superior $y$, en los pocos lugares donde se informa, los resultados son pobres ${ }^{11}$. Un conocimiento inadecuado de esta autoexploración podría ser una de las causas por las cuales las mujeres tienen un entendimiento negativo del pronóstico del cáncer detectado temprano ${ }^{14}$.

Por lo anterior, es fundamental que tanto profesionales en enfermería como estudiantes de esta carrera tengan conocimientos y prácticas adecuadas sobre el AEM, pues asumen un rol fundamental en la promoción de la salud y prevención de esta patología o alguna otra alteración mamaria $^{2}$. Debido a lo mencionado, este estudio tuvo como objetivo principal analizar los predictores de las prácticas sobre el AEM en estudiantes de Enfermería en una universidad privada de Lima Norte, Perú, 2019 y la hipótesis alterna fue que existen predictores (nivel de conocimientos sobre el AEM y características sociodemográficas y de formación) asociados significativamente a las prácticas sobre el AEM.

\section{METOdOLOGÍA}

El tipo de estudio fue no experimental, siguió un enfoque cuantitativo de corte transversal con un alcance máximo correlacional. La población del estudio estuvo conformada por 207 estudiantes de pregrado de ambos sexos entre 17 a 47 años de edad, de la carrera de Enfermería y de una universidad privada ubicada en el distrito Los Olivos de la ciudad de Lima-Perú, 2019. Se tomó en cuenta a toda la población del estudio, por ende, no se realizó un cálculo muestral para obtener el tamaño de la muestra.

Según los criterios de inclusión del estudio, fueron considerados las personas universitarias de enfermería que hubieran cursado el semestre 2019-II de primer a décimo ciclo. Cabe resaltar que se incluyeron estudiantes de ambos sexos, porque a pesar de que el cáncer de mamaque es más frecuente en las mujeres, los hombres pueden presentarlo también ${ }^{15}$. Incluso estudios revelan que, por la poca frecuencia de casos reportados de cáncer de mama en hombres, ellos no conocen de esta enfermedad y mucho menos de métodos preventivos $^{16,17}$. Por esta razón, los varones no deberían ser ajenos a este procedimiento tanto a nivel personal como profesional. Por otro lado, se excluyeron a las personas estudiantes que no desearon participar y a quienes se retiraron de la universidad en el transcurso del ciclo. Por ello, la población se redujo a 92 participantes.

Para el estudio se tomaron en cuenta como variable dependiente las prácticas sobre el AEM, siendo medida por la realización y la técnica del AEM orientada por el Ministerio de Salud del Perú y el Instituto Nacional de Enfermedades Neoplásicas ${ }^{19}$. La realización de esta variable fue medida por la siguiente pregunta: ¿se ha realizado el autoexamen de mamas en los últimos 6 meses? (variable cualitativa nominal dicotómica no y sí). En cuanto a la técnica del AEM, esta fue medida a través de una guía de observación con 12 pasos. Si la persona participante realizaba correctamente un paso obtenía un punto, de lo contrario, cero (variable 
cuantitativa discreta con un rango de 0 a 12 puntos). La construcción de los ítems se hizo a partir de la literatura $y$, posteriormente, fue validada por $^{18,19}$ cinco jueces expertos, de esta valoración obtuvo un coeficiente de $\mathrm{V}$ de Aiken de 0.99. Posterior a esto, se realizó el coeficiente de alfa de Cronbach, en el cual se obtuvo un 0.98 .

La variable independiente principal fue conocimientos sobre el AEM. Esta fue definida como la información adquirida de la experiencia de cuidado de la salud al realizar la palpación e inspección de las mamas ${ }^{4,20}$. Esta variable fue medida por un cuestionario autodirigido de 11 preguntas, el cual fue evaluado por cinco jueces expertos con un coeficiente de $\mathrm{V}$ de Aiken de 0.98 y un alfa de cronbach de 0.96. Se clasificó como una variable cualitativa ordinal politómica (bajo, medio y alto), con los indicadores de 1-3, 4-7 y 8-11 respectivamente.

En relación con las otras variables independientes, se agruparon en características sociodemográficas y de formación ${ }^{8,11,21,22}$. Las sociodemográficas fueron edad en años, variable cuantitativa discreta; sexo de la persona, variable dicotómica (femenino y masculino); lugar de procedencia, variable cualitativa politómica (costa, sierra, selva y extranjero); tipo de seguro, variable politómica (seguro integral de salud, EsSalud, privado y ninguno). Las de formación fueron año académico, variable ordinal politómica (primer año, segundo año, tercer año, cuarto año y quinto año); capacitación, medida a través de la pregunta: ¿ha recibido alguna capacitación sobre detección temprana del cáncer de mama ${ }^{22}$, variable cualitativa dicotómica (no y sí) y estudios técnicos en enfermería, variable cualitativa dicotómica (no y sí).

\section{Recolección de datos}

Para recolectar los datos se les pidió a las personas universitarias participantes que asistieran un día programado al laboratorio de enfermería. Respecto a la medición de la técnica del AEM, se utilizaron herramientas como un espejo, una camilla, una almohada y un biombo para tener privacidad. La técnica de recolección de datos fue la observación, además, se aplicó como instrumento una ficha de cotejo con 12 pasos para verificar si los cumplía o no. En estos pasos se verificó si la persona universitaria, frente al espejo con los brazos hacia abajo y a los costados, observaba sus mamas detalladamente $y$ había buscado algún nódulo, cambios en el pezón, en la forma y tamaño de las mamas. Luego, se evaluó la colocación firme de las manos en las caderas, pero observando sus mamas, así como con los brazos extendidos hacia arriba. Al terminar, se verificó la colocación de la mano izquierda detrás de la cabeza donde la persona debería examinarse la mama izquierda con la mano derecha, palpando con las yemas de los dedos en sentido de la aguja del reloj desde la axila hasta llegar al pezón, con el fin de inspeccionar la existencia de alguna secreción. Luego se verificaba nuevamente este paso, pero con la otra mano. Finalmente, se verificó que la persona estudiante se colocara en una camilla y se palpara con sus manos las mamas en esa posición, explorando con la mano izquierda su mama derecha y repitiendo la misma operación con la mama izquierda. Esta evaluación se efectuó en 8 minutos aproximadamente por persona durante los meses de noviembre y diciembre de 2019.

\section{Análisis de datos}

Se utilizó el paquete estadístico Stata SE versión 14 , se calcularon las frecuencias y los porcentajes para las variables cualitativas, así como las medias aritméticas y desviaciones estándar de las variables cuantitativas. En un inicio, se realizó la prueba de Kolmogórov-Smirnov para comprobar si las variables cuantitativas se ajustan a una distribución normal en los grupos de comparación $(p>.05)$. Luego, se verificó con la prueba de Levene la 
homocedasticidad de varianzas. Comprobados estos requisitos, se realizaron las pruebas de $t$ de Student y ANOVA para la técnica del AEM. Asimismo, se usó la U de Mann Whitney y Kruskal-Wallis por la edad. Del mismo modo, se usaron el chi-cuadrado y Spearman para los análisis bivariados. Se estimó la tendencia de colinealidad mediante el factor de inflación de la varianza y la prueba de Hosmer-Lemeshow para el diagnóstico de regresión. Para determinar los predictores, se realizó el análisis multivariado donde se emplearon la regresión lineal y la de Poisson con la estimación robusta usando la razón de prevalencia $(P R)^{23}$, considerándose los intervalos de confianza al $95 \%$ y un nivel de significancia menor o igual a .05 .

\section{Consideraciones éticas}

El presente trabajo fue registrado en el Departamento de Investigación de la Facultad de Ciencias de la Salud con el código de R620, asimismo fue aprobado por el Comité de Ética de Investigación en Salud de la Universidad Católica Sedes Sapientiae con el código CE 538. Se explicó el objetivo de la investigación a las personas estudiantes en el consentimiento informado, se garantizó la confidencialidad de la información y el anonimato de las personas participantes, para que pudieran responder de manera voluntaria y sin coacciones. Finalmente, las características del estudio no pusieron en riesgo la salud de quienes participaron.

\section{RESULTADOS}

En la tabla 1 se describen las características sociodemográficas y de formación académica de las personas estudiantes de enfermería que participaron. La edad media fue de 24 años, el $23.91 \%$ ha recibido alguna capacitación sobre detección temprana del cáncer de mama. Asimismo, en la tabla 2 se observa que el $67.39 \%$ tuvo un conocimiento medio sobre el AEM, el $35 \%$ realizó el
AEM y la técnica del AEM fue baja $(M=4.41$ de un rango de 0 a 12 puntos).

\section{Tabla 1}

Características sociodemográficas y de formación de estudiantes de enfermería de una universidad privada de Lima, 2019

\begin{tabular}{|c|c|c|}
\hline & fi & (\%) \\
\hline \multicolumn{3}{|c|}{ Características sociodemográficas } \\
\hline Edad (media $\pm \mathrm{DE}$ ) & \multicolumn{2}{|c|}{$24.22 \pm 7.42$} \\
\hline \multicolumn{3}{|l|}{ Sexo } \\
\hline Femenino & 82 & 89.13 \\
\hline Masculino & 10 & 10.87 \\
\hline \multicolumn{3}{|l|}{ Lugar de procedencia } \\
\hline Costa & 73 & 79.35 \\
\hline Sierra & 17 & 18.48 \\
\hline Selva & 1 & 1.09 \\
\hline Extranjero & 1 & 1.09 \\
\hline \multicolumn{3}{|l|}{ Tipo de seguro } \\
\hline Seguro integral de salud & 28 & 30.43 \\
\hline EsSalud & 27 & 29.35 \\
\hline Privado & 5 & 5.43 \\
\hline Ninguno & 32 & 34.78 \\
\hline \multicolumn{3}{|l|}{ Características de formación } \\
\hline \multicolumn{3}{|l|}{ Año académico } \\
\hline Primer año & 26 & 28.16 \\
\hline Segundo año & 17 & 18.48 \\
\hline Tercer año & 16 & 17.39 \\
\hline Cuarto año & 22 & 23.91 \\
\hline Quinto año & 11 & 11.96 \\
\hline \multicolumn{3}{|c|}{$\begin{array}{l}\text { Capacitación sobre detección } \\
\text { temprana del cáncer de mama }\end{array}$} \\
\hline No & 70 & 76.09 \\
\hline Sí & 22 & 23.91 \\
\hline \multicolumn{3}{|c|}{ Estudios técnicos en enfermería } \\
\hline No & 74 & 80.43 \\
\hline Sí & 18 & 19.57 \\
\hline
\end{tabular}

Nota: Frecuencia absoluta (fi), porcentaje (\%) y desviación estándar (DE).
Por otro lado, se reportó una relación positiva entre la realización y la técnica del AEM con el nivel 
de conocimientos sobre el AEM $(p<0.05)$, edad en años $(p<0.001)$, año académico $(p<0.05)$ y estudios

\section{Tabla 2}

Descripción de las prácticas y conocimientos sobre autoexamen de mama de estudiantes de enfermería de una universidad privada de Lima, 2019

\begin{tabular}{llc}
\hline & $\mathbf{f i}$ & $\%$ \\
\hline $\begin{array}{l}\text { Realización del autoexamen de } \\
\text { mamas }\end{array}$ & & \\
$\quad$ No & 60 & 65.22 \\
$\quad$ Sí & 32 & 34.78 \\
$\begin{array}{l}\text { Técnicas del autoexamen de } \\
\text { mamas (media } \pm \text { DE) }\end{array}$ & \multicolumn{2}{|c}{$4.41 \pm 2.12$} \\
Nivel de conocimientos sobre el & & \\
autoexamen de mamas & & \\
$\quad$ Bajo & 12 & 13.04 \\
$\quad$ Medio & 62 & 67.39 \\
$\quad$ Alto & 18 & 19.57 \\
\hline
\end{tabular}

Nota: Frecuencia absoluta (fi), porcentaje (\%) y desviación estándar (DE).

técnicos en enfermería $(p<0.05)$. Sin embargo, el que hubieran recibido capacitación sobre detección temprana del cáncer de mama se relacionó significativamente con la realización del AEM $(p<0.001)$ (ver tabla 3$)$.

Se realizaron diagnósticos de posestimación de los modelos, donde la bondad de ajuste fue mayor a 0.05 y el factor de inflación de la varianza fue de 1.65 (para constatar la existencia de multicolinealidad). Para ello, se ajustaron el año académico y estudios técnicos de enfermería para cumplir los criterios antes mencionados. Se encontró una relación directa entre el nivel de conocimiento sobre el AEM y la realización del $A E M$, de modo que las personas estudiantes con un nivel de conocimiento alto demostraron mayor probabilidad de realizarse el AEM (PRa = 8.73; IC: 1.37-55.8; $p=0.022$ ), respecto a las de nivel bajo. Las personas estudiantes que han recibido capacitación sobre sobre detección temprana del cáncer de mama presentaron mayor probabilidad de realizarse el $\mathrm{AEM}$ ( $\mathrm{PRa}=2.42$; IC: 1.31-4.49; $p=0.005$ ) que quienes que no recibieron. Asimismo, la técnica del AEM aumentó significativamente al incrementarse el nivel de conocimiento sobre el AEM (Coef.a = 1.709; IC: 0.233.18; $p=0.023$ ). Por último, la edad tuvo efectos significativos con la realización del AEM (PRa = 1.04; IC: $1.00-1.07 ; p=0.021$ ) y la técnica del AEM (Coef.a: 0.087; IC: 0.02-0.15; $p=0.008$ ). El resto de los resultados se puede observar en la tabla 4.

\section{DISCUSIÓN}

Un hallazgo importante del estudio fue que el nivel de conocimiento sobre el AEM con mayor porcentaje fue el medio (67.39\%). Un estudio realizado en estudiantes de enfermería de la provincia de IcaPerú mostró que el conocimiento fue medio $(57.14 \%)^{11}$. Por otro lado, en un estudio realizado en la capital de Colombia, el $92.80 \%$ de estudiantes de enfermería no conocía esta técnica preventiva ${ }^{8}$. Asimismo, otro estudio realizado en Turquía mostró el mismo resultado ${ }^{24}$. Esto podría explicarse porque el nivel de conocimiento suele ser mediado por la existencia y la ubicación del contenido sobre AEM en el plan de estudios. Es decir, este contenido se brinda aproximadamente a partir del 3 año académico, por ende, naturalmente el conocimiento puede ser menor en estudiantes de primero y segundo año, por lo tanto, el promedio resultaría medio.

Además, específicamente en hombres, algunas investigaciones descriptivas demostraron que para esta población los conocimientos sobre prevención del cáncer de mama son deficientes y la educación que se les brinda sobre la autoexploración es baja y desalentadora. Esto concuerda con la poca información existente para la mortalidad, estadio y 
Tabla 3

Asociación de las prácticas con el nivel de conocimientos sobre el autoexamen de mamas y las características sociodemográficas y de formación de estudiantes de enfermería de una universidad privada de Lima, 2019

Prácticas sobre el autoexamen de mamas

Realización del autoexamen de mamas

Técnicas del autoexamen de

mamas

\begin{tabular}{llllll}
\hline No & Sí & & & & \\
\hline fi (\%) & fi (\%) & $p$ & Media & DE & $p$
\end{tabular}

Nivel de conocimientos sobre el

autoexamen de mamas

$\begin{array}{ll}\text { Bajo } & 11(91.67) \\ \text { Medio } & 44(70.97) \\ \text { Alto } & 5(27.78)\end{array}$

Características sociodemográficas

Edad en años (m $\pm D E)$

Sexo

Masculino

$22.33 \pm 5.81$

Femenino

$9(90.00)$

$51(62.20)$

Lugar de procedencia

Costa

Sierra

Selva

Extranjero

$45(61.64)$

13 (76.47)

1 (100.00)

$<0.001$

0.023

Tipo de seguro

Seguro integral de salud

EsSalud

$1(100.00)$

$1(8.33)$
$18(29.03)$
$13(72.22)$

3.8

2.04

4.2

2.00

5.6

2.25

Privado

Ninguno

Características de formación

Año académico

Primer año

Segundo año

Tercer año

Cuarto año

Quinto año

$27.75 \pm 8.81$
$1(10.00)$
$31(37.80)$
$28(38.36)$
$4(23.53)$
$(0.00)$
$(0.00)$

0.001

$0.426^{\mathrm{a}}$

$<0.001$

0.055

0.571

$\begin{array}{ll}3.2 & 1.93 \\ 4.6 & 2.11\end{array}$

0.489

8 (28.57)

$20(71.43)$

$9(33.33)$

$18(66.67)$

$3(60.00)$

20 (62.50)

$12(37.50)$

4.3

0.435

$\begin{array}{ll}4.8 & 1.07\end{array}$

$3.0 \quad 0.00$

$\begin{array}{ll}7.0 & 0.00\end{array}$

0.072

Capacitación sobre detección temprana del cáncer de mama

$\begin{array}{ll}\text { No } & 54(77.14) \\ \text { Sí } & 6(27.27)\end{array}$

Estudios técnicos en enfermería No Sí

$22(84.62)$
$11(64.71)$
$11(68.75)$
$9(40.91)$
$7(63.64)$

$53(71.62)$

7 (38.89)

$\begin{array}{ll}3.9 & 1.84 \\ 4.1 & 2.04 \\ 5.8 & 3.63 \\ 4.9 & 2.02\end{array}$

0.038

$<0.001$

$4(15.38)$
$6(35.29)$
$5(31.25)$
$13(59.09)$
$4(36.36)$

3.0

1.65

4.2

1.81

$4.6 \quad 1.93$

$5.7 \quad 2.44$

$\begin{array}{ll}5.4 & 1.03\end{array}$

$<0.001$

0.3653

$16(22.86)$
$16(72.73)$

4.3

2.16

4.8

2.00

0.009

0.015

$4.2 \quad 2.01$

5.5

2.28

Nota: Frecuencia absoluta (fi), porcentaje (\%), desviación estándar (DE), p-valor (p), ${ }^{a}$ Fuerza de correlación 
Tabla 4

Asociación entre el nivel de conocimiento, edad y capacitación con mayor prácticas sobre el autoexamen de mamas estudiantes de enfermería de una universidad privada de Lima, 2019

Prácticas sobre el autoexamen de mamas

Realización del autoexamen de mamas

Técnicas del autoexamen de mamas

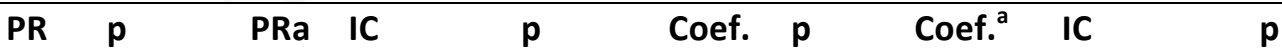

Nivel de conocimientos sobre el

autoexamen de mamas

Bajo

Medio

Ref.

Ref.

Ref. - Ref.

Alto

$\begin{array}{ll}3.48 & 0.204\end{array}$

2.85

$0.46-17.6 \quad 0.261$

0.344

0.597

0.043

$-1.22-1.30$

0.945

$\begin{array}{ll}8.67 & 0.027\end{array}$

$\begin{array}{lll}8.73 & 1.37-55.8 & 0.022\end{array}$

1.778

0.023

1.709

0.23-3.18

0.023

Características sociodemográficas

Edad en años

$\begin{array}{lllll}1.05 & <.001 & 1.04 & 1.00-1.07 & 0.021\end{array}$

$\begin{array}{lll}0.073 & 0.014 & 0.087\end{array}$

$0.02-0.15$

0.008

Características de formación

Capacitación sobre detección

temprana del cáncer de mama

No

Sí

Ref.

Ref.

Ref.

Ref.

${ }^{a}$ ajustado con año académico y estudios técnicos.

diagnóstico, así como a la poca importancia que se la esta enfermedad en los varones por ser poco frecuente, pero existente. Asimismo, por aspectos normativos, en algunos países se ha priorizado el AEM exclusivamente para mujeres, lo cual responde a los aspectos sociales relacionados con esta patología, de cómo se entiende culturalmente, se vive y se atiende ${ }^{16,17,22}$.

Respecto a las prácticas del AEM, fueron medidas por dos variables, en la primera resultó que el 65.22 \% refirió no practicarlo en los últimos 6 meses. Algunos estudios de otros países concuerdan con este hallazgo ${ }^{8,24}$; sin embargo, en otros países se mostró lo contrario. Un gran porcentaje de estudiantes de Enfermería que reportó haberlo practicado $^{13,25}$.

En el resultado anterior, los estudiantes participantes refirieron si se practicaron este procedimiento, pero para constatar si lo hicieron correctamente se midió otra variable titulada la técnica del AEM. Esta variable fue medida a través de una serie de pasos evaluados mediante la técnica de observación por parte de las personas autores. De lo anterior, el promedio resultó bajo en los estudiantes limeños. Una investigación realizada en Perú mostró un resultado similar ${ }^{11}$, mientras que otro estudio en Colombia mostró lo contrario ${ }^{13}$.

Los hallazgos anteriores se pueden relacionar con el hecho de que en la mayoría de los países en vías de desarrollo no se da suficiente importancia y priorización a las políticas públicas de promoción de la salud y prevención de la enfermedad ${ }^{26}$. Asimismo, esta discrepancia se debe a que la mayoría de las personas participantes del estudio no tuvo la oportunidad de capacitarse en esta técnica ${ }^{6}$. Incluso se mostró poco interés por parte de los participantes varones en capacitarse y practicar la técnica, lo cual se explica por la menor exposición al tema del AEM y porque no consideran que esta técnica sea de utilidad para ellos por ser menos propensos al cáncer 
de mama ${ }^{22}$. Por esta razón, las autoridades de la universidad y, en especial, el programa de estudio de Enfermería deben priorizar que las personas estudiantes, en su formación académica, adquieran la información necesaria sobre técnicas preventivas tanto en mujeres como hombres.

Un resultado importante fue que la variable conocimientos sobre el AEM se asoció significativamente con la práctica de este, es decir las personas estudiantes con un nivel alto de conocimiento tuvieron ocho veces más posibilidades de practicarlo en los últimos 6 meses que quienes tienen un nivel bajo de conocimientos. Asimismo, respecto a la técnica sobre el AEM, las personas estudiantes con un buen conocimiento sobre este procedimiento tuvieron mayor probabilidad de practicar una mejor técnica. En la literatura no se reportaron resultados similares realizados específicamente en estudiantes de ambos sexos de Enfermería, sin embargo, se encontró similitud con una investigación realizada en Colombia. En esta, las universitarias de ciencias de la salud que tenían conocimientos suficientes sobre el cáncer de seno tuvieron tres veces más posibilidad de practicar medidas preventivas sobre esta enfermedad mensualmente ${ }^{8}$. Además, un estudio realizado en Nigeria reportó que las universitarias de pregrado con buen conocimiento del AEM presentaron seis veces más probabilidades de practicarlo en comparación con quienes tuvieron poco conocimiento9. Por último, se encontró en una investigación que las estudiantes de ciencias de salud de una universidad de Etiopía que tuvieron buen conocimiento sobre el cáncer de seno practicaron el AEM dos veces más que las que tuvieron poco conocimiento $^{10}$.

Esto puede explicarse por el hecho de que el conocimiento del AEM es reconocido como un precursor necesario para la realización adecuada de su técnica, además, porque las personas estudiantes de Enfermería crean conciencia, por su misma profesión, que deben tener una educación sanitaria sobre medidas preventivas del cáncer de mama ${ }^{27}$. Por lo contrario, la falta de conocimiento y no educar a las personas estudiantes en este procedimiento se ha identificado como barreras importantes para su respectiva práctica ${ }^{28}$. En efecto, tanto la falta de conocimiento de las personas estudiantes como la necesidad de una mayor educación en salud pública sobre el AEM a través los medios de comunicación afectan negativamente la prevención y el pronto diagnóstico del cáncer de mama.

La teorista Patricia Benner menciona en una de sus investigaciones que el proceso de formación de la persona profesional en enfermería requiere no solo de habilidades y destrezas sino de conocimientos que se acumulan con el tiempo. Esta teoría debe estar aplicada o reflejada en las prácticas tanto en las personas estudiantes (principiante) como las profesionales de enfermería. Es por eso que, cuando una persona profesional en enfermería no realiza una adecuada práctica, se relaciona directamente con el aprendizaje que adquirió a lo largo del tiempo ${ }^{29}$.

La edad en años de los estudiantes fue un predictor en las prácticas del AEM tanto en la realización como la técnica. Esto quiere decir que a mayor edad existe más adherencia a la práctica y mejoría en la técnica. Un estudio realizado en mujeres estudiantes de una universidad pública de Malasia evidenció que la edad resultó un predictor de la realización del AEM (OR: 1.3; coeficiente: 0.2$)^{30}$. Esto podría deberse a que las personas toman conciencia de la importancia de la autoexploración de los senos a través de los años, posiblemente por la experiencia en la vida académica o porque la prevalencia del cáncer de mama está relacionada con la edad ${ }^{31}$.

Se halló una asociación entre la realización del AEM en los últimos 6 meses con la variable capacitación. Las personas estudiantes que 
recibieron capacitación sobre la detección temprana del cáncer de mama tienen dos veces más probabilidades de realizarse el AEM en comparación de quienes no se capacitaron. En la literatura no se hallaron resultados similares realizados en estudiantes de enfermería de ambos sexos, sin embargo, se encontró similitud con un estudio realizado en Turquía, donde las universitarias de distintas facultades que fueron educadas en algún curso relacionado al AEM lo practicaron ${ }^{7}$. Además, se encontró en una investigación donde las estudiantes de distintas facultades de Etiopía que escucharon sobre el cáncer de mama practicaron el AEM ${ }^{27}$. Esto se debe a que las capacitaciones pueden llegar a cambiar de manera positiva el pensamiento de una persona, haciendo que esta reciba conocimientos sobre algún tema para que posteriormente pueda practicarlo y tomarlo como un hábito. En línea con esto, las personas estudiantes de enfermería que recibieron alguna capacitación o participaron en alguna charla sobre saben cómo y cuándo realizarse esta práctica, ${ }^{7}$.

Entre las fortalezas del estudio se destaca la participación tanto de mujeres como hombres, porque el cáncer de mama se puede presentar en ambos sexos. Asimismo, porque los estudiantes hombres también participan en la promoción, prevención y detección temprana de enfermedades, como el cáncer de mama y, por ende, deben tener los conocimientos necesarios para prevenir esta patología y realizar intervenciones educativas a toda la población. Actualmente, hay una escasa evidencia científica sobre la medición de estas variables, especialmente en los varones.

Respecto a las limitaciones del estudio están relacionadas con la poca población que deseó participar y con los sesgos de información y temporalidad que presentan el diseño de corte transversal. Los resultados siguen siendo preliminares pero prometedores y se requiere de estudios a gran escala para su fortalecimiento, así como la creación de otros estudios experimentales que incorporen otras variables; por ejemplo, predictores de cáncer de mama, como los antecedentes familiares, la realización de capacitaciones del AEM a personas estudiantes y su evaluación por parte de docentes, otras carreras de salud, solo a los varones, etc. Actualmente, en Latinoamérica hay un gran reto con el cáncer de mama y su detección temprana, ya que está reglamentada desde diferentes leyes en cada país, y, en algunos casos, solo se estipula y prioriza la práctica del AEM exclusivamente en la población femenina.

\section{CONCLUSIÓN}

Se concluye que los conocimientos sobre el AEM en las personas estudiantes de Enfermería de pregrado son regulares; sin embargo, un punto para reflexionar es su práctica, pues no se realizaba suficiente. Respondiendo al objetivo e hipótesis, los predictores que favorecen tener adecuadas prácticas del AEM son alto conocimiento sobre el procedimiento, asistir a capacitaciones sobre detección temprana del cáncer de mama y tener más años de vida.

Esto demuestra que existe la necesidad de aumentar el conocimiento del AEM en estudiantes de Enfermería, donde se enfatice tanto en la concienciación de este procedimiento como en su práctica continua. Por ello, es necesario detectar, corregir y educar a las personas estudiantes desde la universidad para que tengan una formación más sólida y puedan participar en un futuro en la promoción de la salud en una comunidad. Una recomendación sería incentivar y capacitar a las personas estudiantes como educadoras para estudiantes de ciclos inferiores. Además, se espera que los resultados de esta investigación proporcionen datos para la carrera profesional de 
Enfermería de la universidad donde se realizó el estudio, así como para incluir contenido del AEM en sus programas de salud e incrementar el conocimiento, la actitud y la práctica sobre medidas preventivas de enfermedades en sus estudiantes.

\section{DECLARACIÓN DE CONFLICTO DE INTERESES}

Los autores declaran no tener ningún conflicto de intereses.

\section{REFERENCIAS BIBLIOGRÁFICAS}

1. Organización Panamericana de la Salud. Cáncer de mama [Internet]. Washington: OPS; 2019 [citado el 4 de octubre de 2019]. Disponible en: https://www.paho.org/hq/index.php?option=com _content\&view=article\&id=5041:2011-breastcancer\&Itemid=3639\&lang=es

2. Organización Mundial de la Salud. Enfermería [Internet]. Ginebra: OMS; 2019 [citado el 4 de octubre de 2019]. Disponible en: https://www.who.int/topics/nursing/es/

3. Instituto Nacional de Enfermedades Neoplásicas. Casos nuevos de cáncer registrados en INEN, periodo 2009-2018 [Internet]. Lima: INEN; 2019 [citado el 28 de junio de 2020]. Disponible en: https://portal.inen.sld.pe/wpcontent/uploads/2019/12/INEN-2009-2018.pdf

4. Organización Mundial de la Salud. Cáncer de mama: prevención y control [Internet]. Ginebra: OMS; 2009 [citado el 4 de octubre de 2019]. Disponible en: https://www.who.int/topics/cancer/breastcancer /es/index1.html

5. Torres J, Arce MA, Ruiz EF, Mejía CR. Actitudes frente a la autoexploración de mama en dos facultades de ciencias de la salud de Ica, Perú: un estudio piloto. Salud Pública de México, 2017; 59 (2):117-118. DOI: https://doi.org/10.21149/7883

6. Getu MA, Kassaw MW, Tlaye KG, Gebrekiristos AF. Assessment of breast self-examination practice and its associated factors among female undergraduate students in Addis Ababa University, Addis Ababa, Ethiopia, 2016. Dove Medical Press, 2018; 2019 (11): 21-28. DOI: https://doi.org/10.2147/BCTT.S189023

7. Basaran O, Uskun E, Erturk C, Savas P. Health beliefs, behaviour and determining factors in breast self - examination among a group of university students. Marmara Medical Journal, 2019; 32(3): 130-136. DOI: http://doi.org/10.5472/marumj.638115

8. Meneses JF, Martìnez J, Chavarriaga MC, González $K$, Vinaccia $S$, Rincòn $A D$, et al. Creencias $y$ conocimientos relacionados a la práctica del autoexamen de mama en mujeres universitarias de Colombia: Un estudio descriptivo. Rev. chil. obstet. Ginecol, 2018; 83(2): 120-129. DOI: http://dx.doi.org/10.4067/s0717-

75262018000200120

9. Ossai EN, Azuogu BN, Ogaranya IO, Ogenyi Al, Enemor DO, Nwafor MA. Predictors of practice of breast self-examination: a study among female undergraduates of Ebonyi State University, Abakaliki, Nigeria. Níger J Clin Pract, 2019; 22 (3): 361-369.

http://dx.doi.org/10.4103/njcp.njcp_482_18

10. Kassa RT, Wakira HT, Gebremariam MB, Tullu SA, Shehissa NK. Breast Cancer Knowledge and Breast Self-examination Practice among Female Students in Rift Valley University, Adama campus, Adama, Ethiopia, 2017. Journal of Women's Health Care, 2017; $6 \quad$ (5): $\quad 1-7 . \quad$ DOI: http://dx.doi.org/10.4172/2167-0420.1000399

11. Urure IN, Pacheco LA. Conocimientos, actitudes y prácticas sobre autoexamen de mama en estudiantes de enfermería en una universidad pública de la ciudad de Ica, octubre 2017setiembre 2018. Revista Enfermería la Vanguardia, 2019; $7 \quad$ (1): $\quad 3-12 . \quad$ DOI: https://doi.org/10.35563/revan.v7i1.185 
12. Romli HA, Pardi KW. Knowledge, attitude and practice of breast self-examination among undergraduate health science female students. International Journal of Public Health and Clinical Sciences, 2017; 4 (5): 87-99. Disponible en: http://publichealthmy.org/ejournal/ojs2/index.ph p/ijphcs/article/view/487

13. Bohórquez C, Castillo I, Montalvo A. Autoexamen de mama en estudiantes de enfermería de Cartagena, Colombia. Archivos de Medicina, 2017; 17 (2): 361-368. DOI: https://doi.org/0.30554/archmed.17.2.2040.2017

14. Ahmed A, Zahid I, Ladiwala ZR, Sheikh R, Memon AS. Breast self-examination awareness and practices in young women in developing countries: A survey of female students in Karachi, Pakistan. Journal of Education and Health Promotion, 2018; 7: 90. DOI: https://doi.org/10.4103/jehp.jehp_147_17

15. Angel J, Ibarra J, Diaz S, Lehmann C, Garcia M, Guzman L, et al. Comportamiento clínico de cáncer de mama en hombres en una población latinoamericana. Rev Colomb Cancerol, 2015; 19 (3): 150-155.

DOI: https://doi.org/10.1016/j.rccan.2015.05.001

16. Najdyhor E, Krajewska E, Krajewska K. Conocimiento de mujeres y hombres sobre la prevención del cáncer de mama. Ginekologia Polska, 2013; 84 (2): 116-125. DOI: 10.17772 / gp / 1551.

17. Velosa LD, Mejía ME. Perfil social, educativo y conocimiento que tiene un grupo de profesionales de enfermería que trabaja en programas de promoción y prevención sobre el cáncer de mama en hombres. Medicina UPB, 2015; 34 (2): 95-101.

18. EsSalud. Detección temprana del cáncer de mama en EsSalud [Internet]. Lima: EsSalud; 2016 [citado el 4 de octubre de 2019]. Disponible en: http://www.essalud.gob.pe/ietsi/pdfs/guias/DIRE C_DETECCION_TEMP_CANCER_MAMA.pdf
19. Coalición Multisectorial "Perú contra el cáncer", Instituto Nacional de Enfermedades Neoplásicas. Norma técnico oncológica para la prevención, detección y diagnóstico temprano del cáncer de mama a nivel nacional [Internet]. Lima: MINSA; 2008 [citado el 4 de octubre de 2019]. Disponible en:

https://bvs.minsa.gob.pe/local/MINSA/1786.pdf

20. Segarra M, Bou JC. Conceptos, tipos y dimensiones del conocimiento: configuración del conocimiento estratégico. Revista de Economía y Empresa, 2005; 22 (53): 175-196. Disponible en: https://dialnet.unirioja.es/servlet/articulo?codigo $=2274043$

21. Barrionuevo L, Palencia L, Borrel C. ¿Cómo afecta el tipo de seguro de salud a la realización del Papanicolaou en Perú? Rev. Panam. Salud Pública, 2013; 34 (6): 393-400. Disponible en: https://www.scielosp.org/pdf/rpsp/v34n6/v34n6a 04.pdf

22. Carrillo RM, Espinoza ME, Avilez J, Osada J. Nivel de conocimiento y frecuencia de autoexamen de mama en alumnos de los primeros años de la carrera de Medicina. Revista Médica Herediana, 2015; 26: 209-216. DOI: https://doi.org/10.20453/rmh.v26i4.2699

23. Espelt A, Marí-Dell'Olmo M, Penelo E, BosqueProus M. Estimación de la Razón de Prevalencia con distintos modelos de Regresión: Ejemplo de un estudio internacional en investigación de las adicciones. Adicciones, 2016; 29 (2): 105-12. Disponible en: https://adicciones.es/index.php/adicciones/article /view/823/776

24. Koc G, Gulen H, Ergol S, Yildirim M, Aydin N. Female university students' knowledge and practice of breast self-examination in Turkey. Níger J Clin Pract, 2019; 22 (3): 410-415. DOI: http://dx.doi.org/10.4103 njcp.njcp_341_18 
25. Haruna H, Chukwu EO, Ahmadu I, Teryila KR, Babaji $\mathrm{M}$, Nelson L, et al. Knowledge and practice of breast self-examination among female nursing students in university of Maiduguri, Borno State, Nigeria. International Journal of Clinical Oncology and Cancer Research, 2017; 2 (3): 57-64. DOI: http://dx.doi.org/10.11648

\section{J.IJCOCR.20170203.12}

26. Rodríguez CR, Padilla GB. Cáncer mamario y cervicouterino: el problema del absentismo femenino en la acción preventiva. Universidad y salud, 2020; 22 (1): 41-51. DOI: http://dx.doi.org/10.22267/rus.202201.173

27. Birhane $K$, Alemaychu $M$, Anawte $B$, Gebremariyam G, Daniel R, Addis S, et al. Practices of breast self-examination and associated factors among female Debre Berhan university students. International Journal of Breast Cancer, 2017; 1-6. DOI: http://dx.doi.org/10.1155 / 2017/8026297

28. Zabadi HA, Ahmad B, Kathem W, Kokash E, Saed $M$, Husean $M$. Knowledge, awareness, and practice of breast self-examination among AnNajah national university female students. Palestinian Medical and Pharmaceutical Journal, 2017; 2 (1): 45-54. Disponible en: https://hdl.handle.net/20.500.11888/3157

29. Benner P. From Novice to Expert. The American Journal of Nursing. 1982; 82 (3): 402-407. Disponible en: https://www.medicalcenter.virginia.edu/therapyservices/3\%20-\%20Benner\%20-

\%20Novice\%20to\%20Expert-1.pdf

30. Akhtari-Zavare M, Juni MH, Irmi ZI, Salmiah MD, Latiffah AL. Health beliefs and breast selfexamination among undergraduate female students in public universities in Klang Valley, Malaysia. Asian Pac J Cancer Prev, 2015; 16 (9): 4019-4023.

DOI:
31. Mariño ER, Rivera E, Padrón ME, García D, Ávalos $\mathrm{MM}$, Fornaris A. Factores de riesgo del cáncer de mama en un consultorio médico. Revista Cubana de Medicina General Integral, 2018; 34 (2): 1-9. Disponible en: http://www.revmgi.sld.cu/index.php/mgi/article/ view/390/186 